\title{
Use of Non-Assigned Interventions in a Randomized Trial of Internet and Telephone Treatment for Smoking Cessation
}

\author{
Caroline O. Cobb PhD ${ }^{1}$, Amanda L. Graham PhD ${ }^{1,2}$ \\ ${ }^{1}$ Schroeder Institute for Tobacco Research and Policy Studies, Legacy, Washington, DC; ${ }^{2}$ Department of Oncology, Georgetown \\ University Medical Center/Cancer Prevention and Control Program, Lombardi Comprehensive Cancer Center, Washington, DC \\ Corresponding Author: Amanda L. Graham, PhD, Schroeder Institute for Tobacco Research and Policy Studies, Legacy, \\ 1724 Massachusetts Avenue, NW, Washington, DC 20036, USA. Telephone: 202-454-5938; Fax: 202-454-5785; \\ E-mail: agraham@legacyforhealth.org \\ Received January 17, 2014; accepted March 25, 2014
}

\begin{abstract}
Introduction: A recent meta-analysis of Internet interventions for smoking cessation found mixed evidence regarding effectiveness. One explanation may be differential use of non-assigned cessation treatments-including other Internet programs-that either amplify or mask study intervention effects. We examined the impact of non-assigned treatment use on cessation outcomes in The iQUITT Study, a randomized trial of Internet and telephone treatment for smoking cessation.
\end{abstract}

Methods: Participants were randomized to a basic Internet $(\mathrm{BI})$ comparison condition $(N=675)$, enhanced Internet $(\mathrm{EI}$ : $N=651$ ), or EI plus telephone counseling (EI+P: $N=679)$. The primary outcome was 30-day point prevalence abstinence (ppa) at 3 and 6 months. Assigned intervention use was assessed with automated tracking data. Assessment of non-assigned treatments included pharmacotherapy, behavioral, alternative, and non-study Internet treatments. Univariate and multivariate logistic regression models examined whether non-assigned treatment use was associated with 30-day ppa.

Results: About $70 \%$ of participants used at least one non-assigned treatment. A higher rate of non-study Internet treatment among BI participants was the only treatment group difference at both 3 and 6 months. Multivariate models controlling for condition and baseline predictors of non-assigned treatment use showed that high-intensity non-study Internet treatment was positively associated with 30-day ppa at 3 and 6 months, and pharmacotherapy and behavioral treatment use was negatively associated with 30-day ppa at 6 months.

Conclusions: Non-assigned treatment use is an important factor to consider when evaluating Internet cessation interventions. Results highlight methodological issues in selecting a comparison condition. Researchers should report non-assigned treatment use alongside main trial outcomes.

\section{INTRODUCTION}

A recent meta-analysis by Civljak, Stead, Hartmann-Boyce, Sheikh, \& Car (2013) found mixed evidence regarding the effectiveness of Internet interventions for smoking cessation. In their review, interactive and individually tailored Internet interventions yielded a statistically significant effect when compared to non-Internet or no-intervention controls, but not when compared to nontailored, noninteractive Internet programs. To advance the science of Internet interventions, it is important not only to understand the mechanisms through which Internet interventions are effective (Ritterband, Thorndike, Cox, Kovatchev, \& Gonder-Frederick, 2009), but also the reasons they do not yield expected effects.

One possible explanation for the mixed results reported by Civljak et al. (2013) may be differential use of other cessation treatments that may amplify or mask study intervention effects. Smokers that participate in web-based cessation studies are often highly motivated to quit smoking (Graham et al., 2011; Richardson et al., 2013; Saul et al., 2007; Zbikowski et al., 2011) and may seek out additional "non-assigned" cessation methods beyond the interventions assigned in the trial. Not unlike participants in other types of studies, those who join Internet cessation trials are free to avail themselves of other cessation interventions. These may include proven methods such as pharmacotherapy and telephone counseling (Fiore, Jaén, Baker, \& Tobacco Use and Dependence Guideline Panel, 2008) or unproven methods such as acupuncture, hypnosis, or products sold on the Internet that "guarantee" an "easy" way to quit smoking. However, unique to web-based cessation trials is the fact that other Internet interventions of varying quality (Bock et al., 2004; Bock, Graham, Whiteley, \& Stoddard, 


\section{Use of non-assigned interventions in a randomized trial}

2008) are literally a click away (Feil, Noell, Lichtenstein, Boles, \& McKay, 2003).

To date, only one web-based cessation study has examined the impact of non-assigned treatment use on cessation outcomes. Danaher, Lichtenstein, McKay, and Seeley (2009) examined 3- and 6-month follow-up data from the Smokers' Health Improvement Program (SHIP), a randomized trial that compared the Quit Smoking Network (QSN) Internet program to the Active Lives control Web site. At 3 months, approximately one quarter of study participants reported using one or more smoking cessation treatment programs that were not explicitly recommended or assigned in their treatment protocol, including pamphlets/books $(12.6 \%)$, other Internet interventions $(9.0 \%)$, hypnotherapy/acupuncture (4.5\%), group counseling $(2.3 \%)$, and individual counseling $(1.7 \%)$. There were no differences in non-assigned treatment use between intervention and control participants. Pharmacotherapy use was recommended in QSN and its use was higher among this group than among control participants $(50.0 \%$ versus $43.8 \%)$. Use of other Internet programs for cessation was the only predictor of 7-day point prevalence abstinence (ppa) at 3 months and repeated ppa at 6 months.

We sought to extend these findings using data from The iQUITT Study (Graham et al., 2011), a three-arm randomized controlled trial of smoking cessation treatment that compared an interactive cessation Web site with a large online social network (enhanced Internet [EI]) alone and in conjunction with proactive telephone counseling (EI plus phone $[\mathrm{EI}+\mathrm{P}])$ against a static, information-only Internet comparison condition (basic Internet [BI]). Study interventions were available for 6 months. Significant short-term effects for the EI+P arm compared to both BI and EI were observed at both 3 and 6 months. However, contrary to a priori hypotheses, the EI arm did not yield higher abstinence rates compared to the BI arm at either timepoint. Using intent to treat analysis, abstinence rates for BI, EI, and $\mathrm{EI}+\mathrm{P}$ (respectively) were $9.1 \%, 10.4 \%$, and $19.0 \%(p<.001)$ at 3 months and $12.2 \%, 14.4 \%$, and $19.7 \%(p<.001)$ at 6 months.

The purpose of these secondary analyses was to explore whether use of non-assigned cessation treatments during the 6-month active intervention period explained these unexpected findings. "Non-assigned" treatments included any cessation intervention other than the Internet and telephone counseling provided in the trial. These analyses sought to: (a) describe the prevalence of assigned and non-assigned treatment use, (b) identify baseline predictors of non-assigned treatment use; (c) examine the relationship between assigned treatment satisfaction and non-assigned treatment use by condition; and (d) assess the effects of non-assigned treatment use on cessation outcomes at 3 and 6 months.

\section{METHODS}

\section{Participants}

Participants in The iQUITT Study were adult smokers ages 18 and older in the United States who smoked five or more cigarettes per day at the time of recruitment (Graham, Bock, Cobb, Niaura, \& Abrams, 2006; Graham et al., 2011). Active user interception sampling (Kaczmirek \& Neubarth, 2005) was used to recruit Internet users who entered the terms quit(ting) smoking, stop(ping) smoking, or smoking in a major Internet search engine and who clicked on a link to the EI intervention being evaluated (www.QuitNet.com). Following online eligibility screening and informed consent and a baseline telephone assessment, participants were randomized to BI $(N=679)$, EI $(N=651)$, or $\mathrm{EI}+\mathrm{P}(N=675)$.

\section{Interventions}

Participants randomized to EI were given 6 months of free access to the premium service of QuitNet. QuitNet is a longstanding, widely used web-based smoking cessation program (Bock, Graham, Cartter, \& Cobb, 2002; Cobb, Graham, Bock, Papandonatos, \& Abrams, 2005; Graham et al., 2006) that incorporates evidence-based elements of tobacco-dependence treatment (Fiore et al., 2008) including practical counseling and tailored information for cessation, recommendations and support for approved pharmacotherapy, and intratreatment social support through a large online social network of current and former smokers (Cobb, Graham, \& Abrams, 2010).

Participants randomized to $\mathrm{EI}+\mathrm{P}$ were offered five proactive calls in a relapse-sensitive schedule (Zhu et al., 1996). Professional telephone counselors established a supportive working relationship with the participant and assisted participants in skill building to prepare for a quit attempt and prevent relapse. Counselors prompted and reinforced use of QuitNet during each call based on real-time summary data regarding a participant's use of the Web site.

Participants randomized to the BI comparison condition were given 6-month free access to a static, information-only Web site comprised of the content on QuitNet. The language, graphics, and formatting of QuitNet were retained in the BI condition, but the interactive features of QuitNet and its social network were not available based on theory-driven hypotheses tested in the parent trial.

\section{Assessment Procedures}

The baseline telephone assessment was administered following online eligibility screening and informed consent. Follow-up assessments were conducted by telephone or online for telephone nonresponders. Follow-up rates at 3 and 6 months were $76.4 \%$ and $74.7 \%$, respectively.

\section{Measures}

There were three sources of data: (a) baseline assessment, (b) automated tracking data extracted from the basic Internet, QuitNet, and telephone databases regarding "assigned" treatment use, and (c) follow-up assessments at 3 and 6 months.

Baseline assessment: Age, gender, race, ethnicity, education, employment, and household income were assessed using standard items (Centers for Disease Control and Prevention, 2002). Self-rated health status (Ware \& Sherbourne, 1992), history of smoking-related illness, and whether they had spoken to a doctor about their smoking were also measured. Smoking variables included cigarettes per day, the time to first cigarette item from the Fagerström Test for Nicotine Dependence (Heatherton, Kozlowski, Frecker, \& Fagerström, 1991), duration of last quit attempt (days), desire to quit and confidence in quitting (scale from 1 to 10). Psychosocial items included the Smoking Situations Confidence Inventory and the Smoking Temptations Inventory (short-form; Velicer, Diclemente, Rossi, \& Prochaska, 1990), Perceived Stress Scale (Cohen, Kamarck, \& Mermelstein, 1983), and the Center for Epidemiological 
Studies Depression Scale (Andresen, Malmgren, Carter, \& Patrick, 1994).

Automated tracking data: Web site utilization metrics were extracted at 3 and 6 months and reflected intervention use since the last follow-up period. Available metrics for both BI and EI included the number of logins, page views, and minutes spent using the intervention. The number of counseling calls completed was extracted at 3 and 6 months for participants randomized to $\mathrm{EI}+\mathrm{P}$.

Follow-up assessment: Smoking outcomes in these analyses included 30-day ppa and the number of intentional quit attempts since the prior assessment period. Among those who logged into their assigned Web site, three intervention satisfaction items assessed overall satisfaction, perceived helpfulness, and how well the intervention met their expectations $(1=$ Not at all, $10=$ Very much).

"Non-assigned" treatment use was assessed at 3 and 6 months only among participants who reported one or more quit attempts. Participants were asked about the use of other cessation Web sites, nicotine replacement therapy (patch, gum, lozenge, spray, and inhaler), prescription medication (e.g., Zyban/bupropion), behavioral treatments (pamphlet/ book, individual counseling, group counseling, telephone counseling), alternative quit methods (e.g., acupuncture, hypnosis, switching to chew/snuff), and any "other" methods which were recorded as text responses and recoded into pharmacological, behavioral, or alternative treatments. Individuals who reported use of other cessation Web sites were asked to identify them and the number of visits $(1-2,3-5,6-10$, more than 10 times).

\section{Statistical Analyses}

First, we examined the proportion of participants in each treatment arm that reported a quit attempt at each follow-up using frequency tables and chi-square tests. Frequencies and descriptive statistics were used to characterize "assigned" and "non-assigned" cessation treatment use. Analysis of variance was used to examine between-group differences in intervention satisfaction among participants who used their assigned Internet program at least once. Non-assigned treatment use was grouped into four categories based upon the U.S. Public Health Service Clinical Practice Guideline (Fiore et al., 2008): pharmacotherapy, behavioral treatments, alternative methods, and Internet treatment. Among participants reporting use of a non-study cessation Web site, we examined intensity of use, with low intensity defined as fewer than three logins and high intensity defined as three or more logins. Analysis of variance and chi-square tests were used to examine treatment group differences on these variables.

Next we examined whether select baseline demographic, smoking, and psychosocial characteristics predicted nonassigned treatment use at each follow-up in a series of multivariate logistic regression models. Any use of pharmacotherapy, behavioral treatments, alternative methods, and non-assigned Internet treatment at 3 and 6 months were entered as outcomes of interest.

Our third set of analyses examined whether ratings of assigned treatment satisfaction at 3 and 6 months were related to non-assigned treatment use by treatment group. Since overall satisfaction was significantly correlated with perceived helpfulness and the extent to which the intervention met expectations at both 3 and 6 months (all Pearson correlations > .80 ), we focus our analyses on overall satisfaction and used a median split to identify participants with low and high levels of treatment satisfaction. Chi-square analyses were used to examine non-assigned treatment use by levels of assigned treatment satisfaction nested within each treatment arm.

Finally, univariate logistic regression models examined whether non-assigned treatment use were associated with 30-day ppa at 3 and 6 months. Use of pharmacotherapy, behavioral quit methods, and alternative quit methods were entered in the model as count variables (i.e., number of methods endorsed); Internet treatment use was examined as a threelevel intensity variable (none, low, high). A similar approach was taken in multivariate models which also included treatment assignment and the covariates identified as predictors of non-assigned treatment use. Finally, we looked at smoking outcomes at each timepoint by treatment arm among participants who reported no non-assigned treatment use.

\section{RESULTS}

\section{Sample Characteristics}

Follow-up rates from the parent study at 3 and 6 months are shown in Figure 1. Among those reached at follow-up, there were no between-group differences in the proportion of participants that reported one or more quit attempts $(90.0 \%$ at 3 months, $73.5 \%$ at 6 months). Among those reporting a quit attempt, complete data on non-assigned treatment use was available at 3 and 6 months for $98.8 \%$ of participants.

\section{Use of Assigned Treatment}

Assigned treatment utilization was the highest during the first 3 months of the study. Approximately 20\%-25\% of participants in each treatment arm never logged into their assigned Web site; there were no differences between groups (Table 1). Among those who did log in, number of logins differed by treatment arm: significantly more participants in $\mathrm{EI}$ and EI+P logged into the site three or more times compared to BI participants $(48.8 \%$ and $48.6 \%$ versus $23.4 \%$, respectively, $p<.001$ ). Between 3 and 6 months, significantly more participants in both EI arms used the site at least once compared to BI participants $(23.8 \%$ and $21.3 \%$ versus $8.2 \%$, respectively, $p<.001$ ) and used the site more intensively $(12.1 \%$ and $10.4 \%$ versus $1.0 \%$ logged in three or more times, respectively; $p<.001)$. Among participants in $\mathrm{EI}+\mathrm{P}, 73.3 \%$ completed at least one call during the 6-month intervention period and the average number of calls completed was $5.1(S D=3.6)$. Phone counseling occurred mostly during the first 3 months of the study: $73.2 \%$ of participants completed at least one call, and the average number of calls was $4.4(S D=2.2)$.

Significant differences by treatment group were observed for all three metrics of intervention satisfaction at both 3 and 6 months, with the lowest ratings consistently observed in the BI arm. Correlations among these three items exceeded Pearson $r=.80$, so for illustration purposes, we present overall satisfaction data here. At 3 months, ratings were 5.7 \pm 2.9 , $7.2 \pm 2.5$, and $7.7 \pm 2.3$ ( $p<.001,10$-point Likert scale) for $\mathrm{BI}, \mathrm{EI}$, and EI+P, respectively. Ratings of overall satisfaction 
Use of non-assigned interventions in a randomized trial

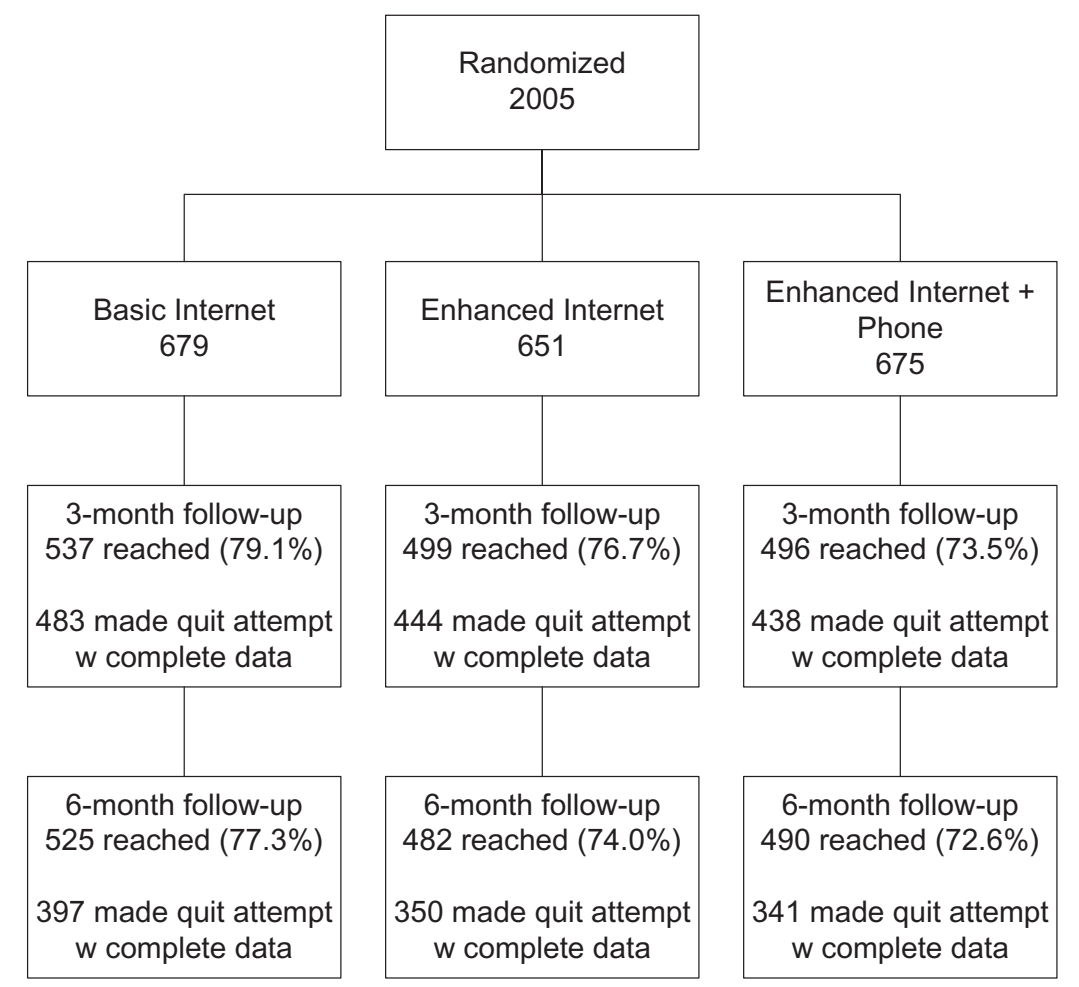

Figure 1. Number and proportion of study participants reached at each follow-up reporting a quit attempt with complete nonassigned treatment utilization data.

were slightly higher at 6 months: $\mathrm{BI}=6.3 \pm 2.7, \mathrm{EI}=7.4 \pm 2.3$, $\mathrm{EI}+\mathrm{P}=7.7 \pm 2.2, p<.001$. The majority of participants in $\mathrm{EI}+\mathrm{P}$ reported the number of counseling calls was "just right" at both 3 months $(81.8 \%)$ and 6 months $(76.1 \%)$.

\section{Use of Non-Assigned Treatment}

Non-assigned treatment use was also highest during the first 3 months, with roughly $70 \%$ of participants in all treatment arms using some form of intervention outside the study (Table 2). About half of participants used one or more forms of pharmacotherapy, about a third used one or more behavioral treatments, and less than $10 \%$ used an alternative quit method. Non-assigned treatment use was slightly lower at 6 months. Greater use of non-assigned Internet Web sites was the only difference observed between treatment arms, with higher rates at both 3 and 6 months in $\mathrm{BI}$ relative to $\mathrm{EI}$ and $\mathrm{EI}+\mathrm{P}(p \mathrm{~s}<.05)$. Intensity of Internet treatment use did not vary significantly across groups at either timepoint.

\section{Baseline Predictors of Non-Assigned Treatment Use}

Greater use of previous quit methods emerged as the most consistent predictor across categories of non-assigned treatment use at both 3 and 6 months (Table 3). At 3 months, predictors of pharmacotherapy use included higher household income ( $\$ 75,000$ or above), time to first cigarette (within $30 \mathrm{~min}$ ), greater use of previous quit methods, and having talked with their doctor about smoking. Predictors of behavioral treatment use included higher levels of education (college or higher), lower likelihood of moderate income $(<\$ 50,000-\$ 74,999)$, and greater use of previous quit methods. No baseline characteristics were significantly associated with use of alternative quit methods. Predictors of non-assigned Internet treatment use included higher levels of education (college or higher) and greater use of previous quit methods.

Many of the same predictors emerged in 6-month models. Predictors of pharmacotherapy use included age, higher level of education (college or higher), higher household income ( $\$ 75,000$ or above), time to first cigarette (within $30 \mathrm{~min}$ ), greater use of previous quit methods, worse health status (very good relative to excellent), and having talked with their doctor about smoking. Predictors of behavioral treatment use included greater baseline smoking rate, time to first cigarette ( $30 \mathrm{~min}$ or more), and greater use of previous quit methods. College education or higher was the only predictor of use of alternative quit methods. Predictors of non-assigned Internet use included higher levels of education (some college or more), greater use of previous quit methods, and greater desire to quit.

\section{Assigned Treatment Satisfaction and Non-Assigned Treatment Use by Treatment Group}

At 3 months, overall satisfaction was related only to nonassigned Internet use. Participants in BI with lower satisfaction were more likely to use other Internet sites compared to those with higher satisfaction $(24.4 \%$ versus $15.0 \%, p=.043)$. This effect was also significant when results were collapsed across treatment group $(18.2 \%$ versus $12.0 \%, p=.004)$. At 6 months, overall satisfaction was related to pharmacotherapy and nonassigned Internet use: participants randomized to EI+P with lower satisfaction were more likely to use pharmacotherapy (54.2\% versus $39.1 \%, p=.037)$ and non-assigned Internet treatment $(13.0 \%$ versus $3.3 \%, p=.024)$ compared to those with higher levels of satisfaction. 

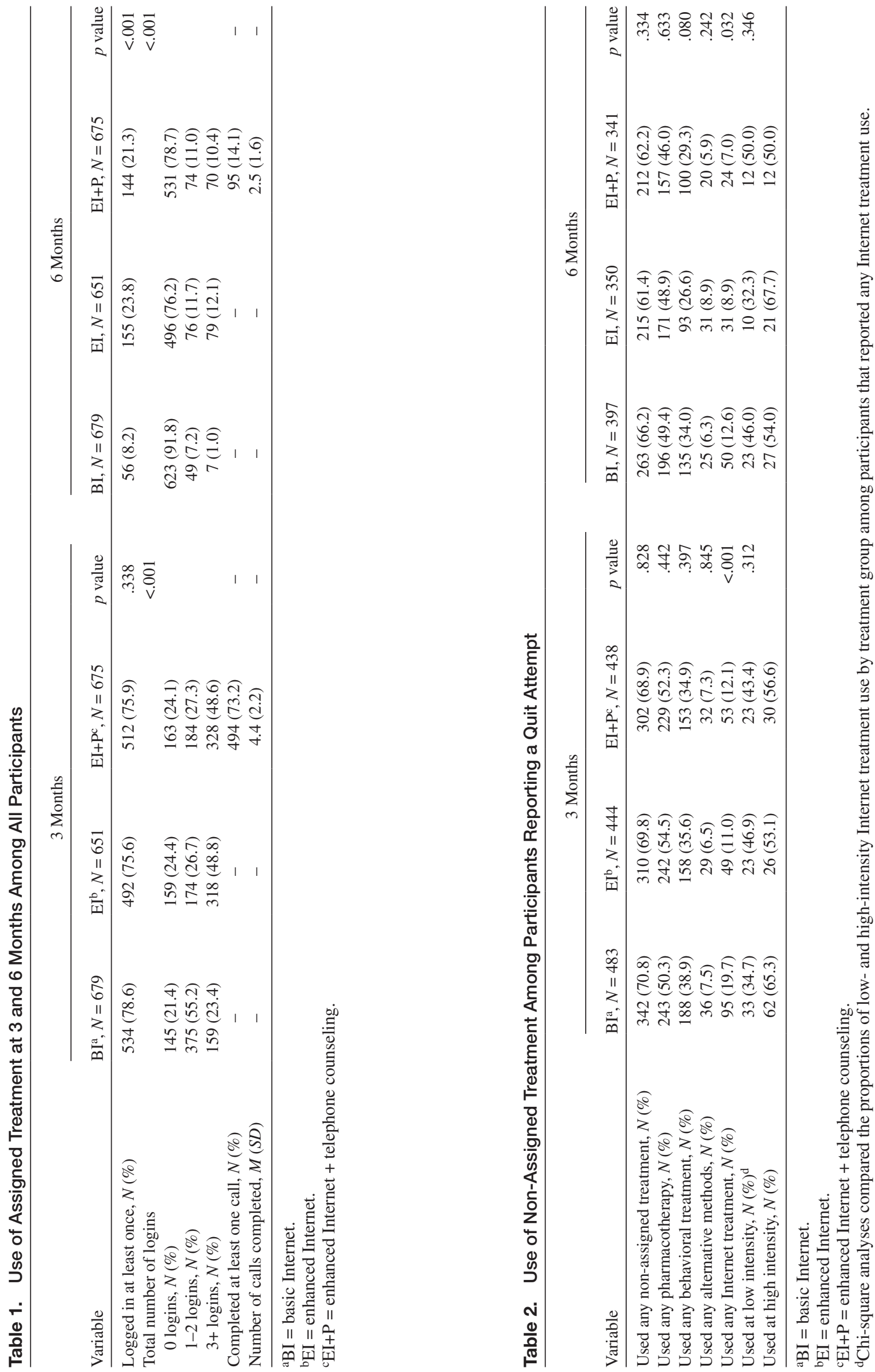


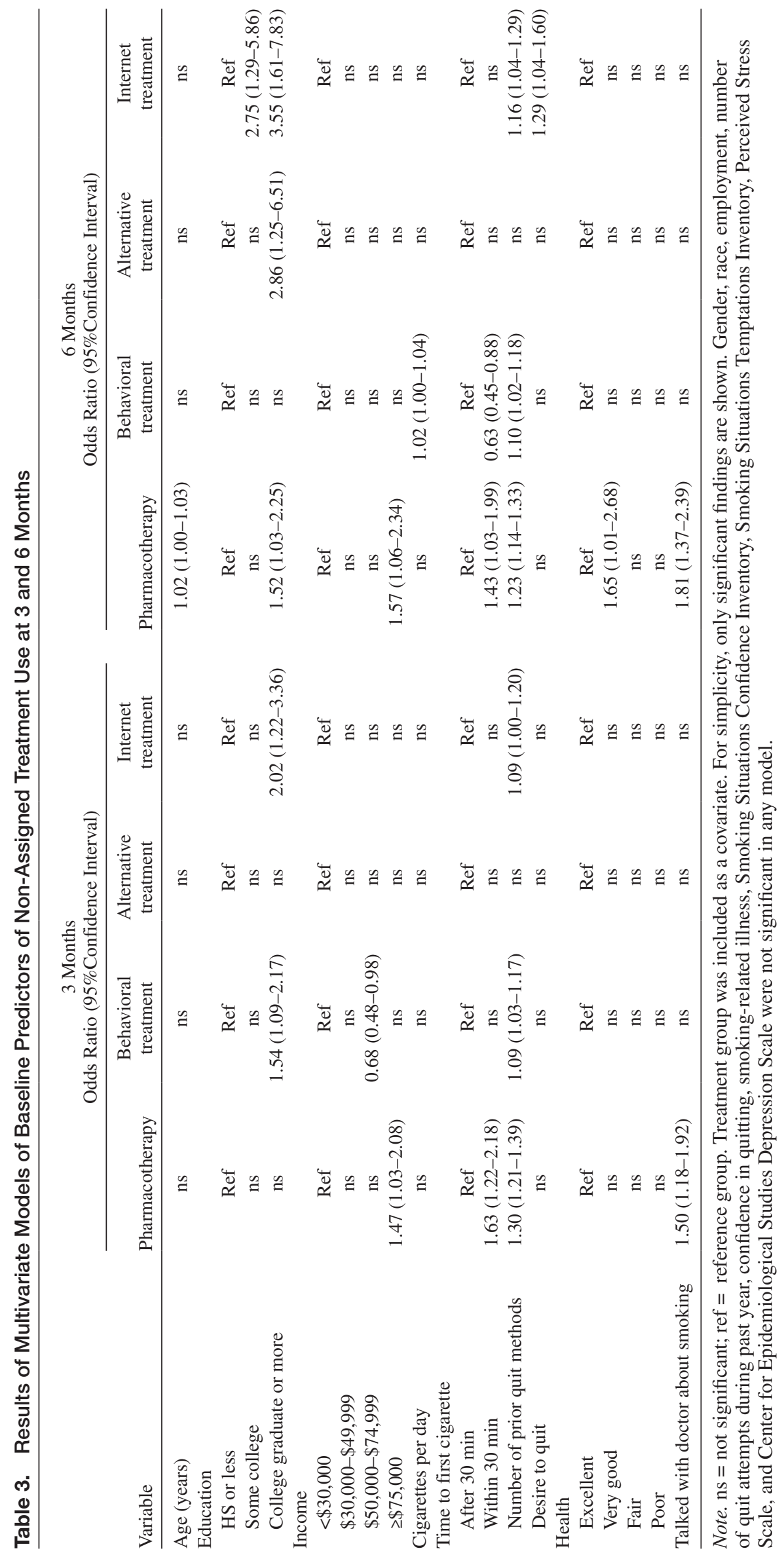




\section{Effects of Non-Assigned Treatment Use on Cessation Outcomes}

Univariate regression models showed a significant effect on 30-day ppa at 3 months for high-intensity non-assigned Internet use (odds ratio $[O R]=1.65,95 \%$ confidence interval $[\mathrm{CI}]=1.07,2.56)$. At 6 months, use of pharmacotherapy $(O R=0.76,95 \% \mathrm{CI}=0.62,0.95)$ and behavioral treatments $(O R=0.72,95 \% \mathrm{CI}=0.56,0.92)$ were associated with lower abstinence rates. These findings persisted in multivariate models. After controlling for treatment assignment and baseline variables associated with non-assigned treatment use (age, education, income, baseline smoking rate, time to first cigarette, previous use of quit methods at baseline, desire to quit, health status, and talked to their doctor about smoking), the only non-assigned treatment method associated with 30-day ppa at 3 months was a high level of non-assigned Internet use which almost doubled the odds of abstinence $(O R=2.10$, $95 \% \mathrm{CI}=1.21,3.65)$. At 6 months, use of pharmacotherapy $(O R=0.75,95 \% \mathrm{CI}=0.59,0.96)$ and behavioral treatment $(O R=0.60,95 \% \mathrm{CI}=0.43,0.83)$ were both related to lower abstinence rates, whereas a high level of Internet use tripled the odds of abstinence $(O R=3.25,95 \% \mathrm{CI}=1.53,6.92)$.

Finally, excluding participants who used any form of nonassigned treatment (approximately $70 \%$ of the sample), 30-day ppa at 3 months was $7.8 \%, 13.4 \%$, and $29.4 \%$ for BI, EI, and EI+P, respectively. At 6 months, 30-day ppa among participants who did not use any form of non-assigned treatment at 3 and 6 months was $10.6 \%, 16.9 \%$, and $24.0 \%$ among BI, EI, and $\mathrm{EI}+\mathrm{P}$, respectively.

\section{DISCUSSION}

The results of these analyses indicate that non-assigned treatment use is common among participants in web-based cessation trials and is an important element to consider in examining cessation outcomes. Across all three intervention arms, nearly $70 \%$ of study participants used one or more quit methods outside the study within the first 3 months of the trial. There were no treatment group differences in the use of pharmacotherapy, behavioral treatments, or alternative quit methods. However, there were significant treatment group differences in the use of non-assigned Internet treatments, with higher rates of use among participants in the BI comparison condition at both 3 and 6 months.

These patterns of non-assigned treatment use are especially interesting when considered in the context of assigned treatment utilization and intervention satisfaction. Although over 75\% from each treatment group accessed their study Web site at least once, participants randomized to BI were significantly less likely to return to the site and reported significantly lower ratings of intervention helpfulness and satisfaction at both 3 and 6 months. These results suggest that a static, informationonly Web site designed to serve as a control condition in a web-based randomized trial-intentionally limited in content and functionality in order to examine the effectiveness of the enhanced Internet intervention-was less engaging to participants and prompted greater use of non-study Internet resources.

Only non-assigned Internet treatment use was significantly related to abstinence at both timepoints in multivariate models that controlled for baseline predictors of use and treatment assignment. These findings are consistent with results from the SHIP trial which also found a positive relationship between use of other Internet programs for smoking cessation and abstinence (Danaher et al., 2009). It is puzzling why other non-assigned treatments-particularly pharmacotherapy use, given its proven effectiveness (Fiore et al., 2008) - were not significantly associated with abstinence. One hypothesis is that use of pharmacotherapy or behavioral treatment is a marker for increased difficulty in quitting. Participants who enroll in a web-based intervention may initially feel that this self-help treatment modality will be sufficient for them to quit, and turn to other forms of intervention when they struggle to achieve abstinence. These questions warrant further investigation. Though the small sample size precluded significance testing, differences in abstinence rates among individuals who did not use any form of non-assigned treatment appeared more pronounced between treatment groups.

These findings have important implications for the selection of a comparison condition in Internet smoking cessation trials. Individuals searching the Internet for cessation assistance likely have some basic expectation of what Web sites should entail and may continue searching to find resources that meet these expectations. Selecting an appropriate comparison condition in web-based trials is an acknowledged challenge (Danaher \& Seeley, 2009; Eysenbach, 2002). A control Web site that contains the content, features, and functionality that users have come to expect on the Internet may be effective in minimizing non-assigned treatment use, but may not differ in substantive ways from study intervention sites.

The challenge in identifying a control condition that minimizes non-assigned treatment use but that also represents an appropriate comparison condition may best be solved by moving away from "horse race" randomized trials and focusing more on innovative research designs specifically constructed to identify the active ingredients and mechanisms through which Internet sites exert their effects on abstinence. Factorial designs are well suited to address questions about which components are most important or effective (Collins, Murphy, \& Strecher, 2007; McClure et al., 2013; Schneider, de Vries, Candel, van de Kar, \& van Osch, 2013). The sequential multiple assignment randomized trial (SMART) may also be useful for testing components of adaptive/time-varying interventions and the sequencing of specific treatment options (Collins et al., 2007). A number of researchers have noted the need for innovative and alternative research designs to systematically and pragmatically assess the potency of individual intervention features (Bennett \& Glasgow, 2009; Danaher \& Seeley, 2009; Sanchez et al., 2013). With this information, future interventions may be able to better leverage this broad-reaching modality for health behavior change.

Our study adds to the results of Danaher et al. (2009) and addresses several of the limitations noted in the SHIP trial. There was an equal representation of men and women in our sample, and the study included multiple assessments of non-assigned treatment use. In addition, follow-up rates at 3 and 6 months were $76.4 \%$ and $74.7 \%$ in The iQUITT Study compared to $44.3 \%$ and $32.8 \%$ in the SHIP trial. Other study strengths include a comprehensive assessment of non-assigned intervention use, the use of automated tracking data to measure assigned intervention use, and the availability of a range of psychosocial and smoking variables included in multivariate models. 
Our findings should be considered in the context of several limitations and in light of our recruitment methods. Participants may have had expectations about the study intervention, perhaps based on its top search engine ranking or exposure to advertising. These expectations may have increased the likelihood of non-assigned Internet use among BI participants, which may not have occurred with a different recruitment approach. Also, it was not feasible to biochemically verify self-reported abstinence since this was a national sample recruited via the Internet. Self-reported abstinence is commonly accepted in web-based cessation trials (Leykin, Aguilera, Torres, Pérez-Stable, \& Muñoz, 2012; Rabius, Pike, Wiatrek, \& McAlister, 2008; Strecher et al., 2008; Wangberg, Nilsen, Antypas, \& Gram, 2011; Zbikowski, Hapgood, Smucker Barnwell, \& McAfee, 2008) where misreporting of abstinence is expected to be minimal (SRNT Subcommittee on Biochemical Verification, 2002).

In conclusion, our data suggest that non-assigned treatment use is an important factor to consider in evaluating the effects of web-based smoking cessation interventions. If completed studies have data on the use of nontrial quit methods, these are important secondary analyses to pursue to get a clearer picture of overall effectiveness of this intervention modality. Future trials should include careful assessment of other treatment utilization and include these analyses alongside main outcomes of the trial.

\section{FUNDING}

This work was supported by the National Cancer Institute of the National Institutes of Health (R01 CA104836). The study is registered at Clinicaltrials.gov (\#NCT00282009).

\section{DECLARATION OF INTERESTS}

COC and ALG are employees of Legacy, a nonprofit public health foundation that runs BecomeAnEX.org, an online tobacco cessation intervention.

\section{REFERENCES}

Andresen, E. M., Malmgren, J. A., Carter, W. B., \& Patrick, D. L. (1994). Screening for depression in well older adults: Evaluation of a short form of the CES-D (Center for Epidemiologic Studies Depression Scale). American Journal of Preventive Medicine, 10, 77-84.

Bennett, G. G., \& Glasgow, R. E. (2009). The delivery of public health interventions via the Internet: Actualizing their potential. Annual Review of Public Health, 30, 273-292. doi:10.1146/annurev.publhealth.031308.100235

Bock, B. C., Graham, A. L., Whiteley, J. A., \& Stoddard, J. L. (2008). A review of web-assisted tobacco interventions (WATIs). Journal of Medical Internet Research, 10, e39. doi:10.2196/jmir.989

Bock, B., Graham, A., Sciamanna, C., Krishnamoorthy, J., Whiteley, J., Carmona-Barros, R., ... Abrams, D. (2004). Smoking cessation treatment on the Internet: Content, quality, and usability. Nicotine \& Tobacco Research: Official Journal of the Society for Research on Nicotine and Tobacco, 6, 207-219. doi:10.1080/14622200410001676332

Bock, B.C., Graham, A.L., Cartter, C., \& Cobb, N. (2002, February 20-24). Smoking Cessation on the Web: Initial
Evaluation of QuitNet. Paper presented at the Society for Research on Nicotine and Tobacco, Savannah, GA.

Centers for Disease Control and Prevention. (2002). 2002 Behavioral Risk Factor Surveillance System - Survey Questions. Atlanta, GA: Centers for Disease Control and Prevention.

Civljak, M., Stead, L. F., Hartmann-Boyce, J., Sheikh, A., \& Car, J. (2013). Internet-based interventions for smoking cessation. The Cochrane Database of Systematic Reviews, 7, CD007078. doi:10.1002/14651858.CD007078.pub4

Cobb, N. K., Graham, A. L., \& Abrams, D. B. (2010). Social network structure of a large online community for smoking cessation. American Journal of Public Health, 100, 12821289. doi:10.2105/AJPH.2009.165449

Cobb, N. K., Graham, A. L., Bock, B. C., Papandonatos, G., \& Abrams, D. B. (2005). Initial evaluation of a realworld Internet smoking cessation system. Nicotine \& Tobacco Research: Official Journal of the Society for Research on Nicotine and Tobacco, 7, 207-216. doi: 10.1080/14622200500055319

Cohen, S., Kamarck, T., \& Mermelstein, R. (1983). A global measure of perceived stress. Journal of Health and Social Behavior, 24, 385-396. doi: 10.2307/2136404

Collins, L. M., Murphy, S. A., \& Strecher, V. (2007). The multiphase optimization strategy (MOST) and the sequential multiple assignment randomized trial (SMART): New methods for more potent eHealth interventions. American Journal of Preventive Medicine, 32(Suppl. 5), S112-S118. doi:10.1016/j.amepre.2007.01.022

Danaher, B. G., Lichtenstein, E., McKay, H. G., \& Seeley, J. R. (2009). Use of non-assigned smoking cessation programs among participants of a Web-based randomized controlled trial. Journal of Medical Internet Research, 11, e26. doi:10.2196/jmir. 1172

Danaher, B. G., \& Seeley, J. R. (2009). Methodological issues in research on web-based behavioral interventions. Annals of Behavioral Medicine: A Publication of the Society of Behavioral Medicine, 38, 28-39. doi:10.1007/ s12160-009-9129-0

Eysenbach, G. (2002). Issues in evaluating health websites in an Internet-based randomized controlled trial. Journal of Medical Internet Research, 4, E17. doi:10.2196/jmir.4.3.e17

Feil, E. G., Noell, J., Lichtenstein, E., Boles, S. M., \& McKay, H. G. (2003). Evaluation of an Internet-based smoking cessation program: Lessons learned from a pilot study. Nicotine \& Tobacco Research: Official Journal of the Society for Research on Nicotine and Tobacco, 5, 189-194. doi:10.1080/1462220031000073694

Fiore, M. C., Jaén, C. R., Baker, T. B., \& Tobacco Use and Dependence Guideline Panel. (2008). Treating Tobacco Use and Dependence: 2008 Update. Clinical Practice Guideline. Rockville, MD: U.S. Department of Health and Human Services. Public Health Service.

Graham, A. L., Bock, B. C., Cobb, N. K., Niaura, R., \& Abrams, D. B. (2006). Characteristics of smokers reached and recruited to an internet smoking cessation trial: A case of denominators. Nicotine \& Tobacco Research, 8(Suppl. 1), S43-S48. doi:10.1080/14622200601042521

Graham, A. L., Cobb, N. K., Papandonatos, G. D., Moreno, J. L., Kang, H., Tinkelman, D. G., ... Abrams, D. B. (2011). A randomized trial of Internet and telephone treatment for smoking cessation. Archives of Internal Medicine, 171, 4653. doi:10.1001/archinternmed.2010.451

Heatherton, T. F., Kozlowski, L. T., Frecker, R. C., \& Fagerström, K. O. (1991). The Fagerström Test for Nicotine Dependence: A revision of the Fagerström Tolerance Questionnaire. British Journal of Addiction, 86, 1119-1127. doi:10.1111/j.1360-0443.1991.tb01879.x 
Kaczmirek, L., \& Neubarth, W. (2005). Improving Web based Intercept surveys: A framework for the active user sampling. Proceedings of The American Association for Public Opinion Research (AAPOR) 60th Annual Conference; West Palm Beach, FL. Retrieved from http://www.websm.org/ main/baza/baza.php?bid=3361\&avtor=619.

Leykin, Y., Aguilera, A., Torres, L. D., Pérez-Stable, E. J., \& Muñoz, R. F. (2012). Interpreting the outcomes of automated internet-based randomized trials: Example of an International Smoking Cessation Study. Journal of Medical Internet Research, 14, e5. doi:10.2196/jmir.1829

McClure, J. B., Shortreed, S. M., Bogart, A., Derry, H., Riggs, K., St John, J., ... An, L. (2013). The effect of program design on engagement with an internet-based smoking intervention: Randomized factorial trial. Journal of Medical Internet Research, 15, e69. doi:10.2196/jmir.2508

Rabius, V., Pike, K. J., Wiatrek, D., \& McAlister, A. L. (2008). Comparing internet assistance for smoking cessation: 13-month follow-up of a six-arm randomized controlled trial. Journal of Medical Internet Research, 10, e45. doi:10.2196/jmir.1008

Richardson, A., Graham, A. L., Cobb, N., Xiao, H., Mushro, A., Abrams, D., \& Vallone, D. (2013). Engagement promotes abstinence in a web-based cessation intervention: Cohort study. Journal of Medical Internet Research, 15, e14. doi:10.2196/jmir.2277

Ritterband, L. M., Thorndike, F. P., Cox, D. J., Kovatchev, B. P., \& Gonder-Frederick, L. A. (2009). A behavior change model for internet interventions. Annals of Behavioral Medicine: A Publication of the Society of Behavioral Medicine, 38, 18-27. doi:10.1007/s12160-009-9133-4

Sanchez, M. A., Rabin, B. A., Gaglio, B., Henton, M., Elzarrad, M. K., Purcell, P., \& Glasgow, R. E. (2013). A systematic review of eHealth cancer prevention and control interventions: new technology, same methods and designs? Translational Behavioral Medicine, 3, 392-401. doi:10.1007/s13142-013-0224-1

Saul, J. E., Schillo, B. A., Evered, S., Luxenberg, M. G., Kavanaugh, A., Cobb, N., \& An, L. C. (2007). Impact of a statewide Internet-based tobacco cessation intervention. Journal of Medical Internet Research, 9, e28. doi:10.2196/ jmir.9.4.e28
Schneider, F., de Vries, H., Candel, M., van de Kar, A., \& van Osch, L. (2013). Periodic email prompts to re-use an internet-delivered computer-tailored lifestyle program: Influence of prompt content and timing. Journal of Medical Internet Research, 15, e23. doi:10.2196/jmir.2151

SRNT Subcommittee on Biochemical Verification. (2002). Biochemical verification of tobacco use and cessation. Nicotine \& Tobacco Research, 4, 149-159. doi:10.1080/14622200210123581

Strecher, V. J., McClure, J. B., Alexander, G. L., Chakraborty, B., Nair, V. N., Konkel, J. M., ... Pomerleau, O. F. (2008). Web-based smoking-cessation programs: Results of a randomized trial. American Journal of Preventive Medicine, 34, 373-381. doi:10.1016/j.amepre.2007.12.024

Velicer, W. F., Diclemente, C. C., Rossi, J. S., \& Prochaska, J. O. (1990). Relapse situations and self-efficacy: An integrative model. Addictive Behaviors, 15, 271-283. doi:10.1016/0306-4603(90)90070-E

Wangberg, S. C., Nilsen, O., Antypas, K., \& Gram, I. T. (2011). Effect of tailoring in an internet-based intervention for smoking cessation: Randomized controlled trial. Journal of Medical Internet Research, 13, e121. doi:10.2196/jmir.1605

Ware, J. E., Jr, \& Sherbourne, C. D. (1992). The MOS 36-item short-form health survey (SF-36). I. Conceptual framework and item selection. Medical Care, 30, 473-483. doi:10.1097/00005650-199206000-00002.

Zbikowski, S. M., Hapgood, J., Smucker Barnwell, S., \& McAfee, T. (2008). Phone and web-based tobacco cessation treatment: Real-world utilization patterns and outcomes for 11,000 tobacco users. Journal of Medical Internet Research, 10, e41. doi:10.2196/jmir.999

Zbikowski, S. M., Jack, L. M., McClure, J. B., Deprey, M., Javitz, H. S., McAfee, T. A., ... Swan, G. E. (2011). Utilization of services in a randomized trial testing phone- and web-based interventions for smoking cessation. Nicotine \& Tobacco Research: Official Journal of the Society for Research on Nicotine and Tobacco, 13, 319-327. doi:10.1093/ntr/ntq257

Zhu, S. H., Stretch, V., Balabanis, M., Rosbrook, B., Sadler, G., \& Pierce, J. P. (1996). Telephone counseling for smoking cessation: effects of single-session and multiple-session interventions. Journal of Consulting and Clinical Psychology, 64, 202-211. doi:10.1037/0022-006X.64.1.202 